\title{
BMC Medical Research Methodology reviewer acknowledgement 2014
}

Giulia Mangiameli

\section{Contributing reviewers}

The editors of BMC Medical Research Methodology would like to thank all our reviewers who have contributed to the journal in Volume 14 (2014).

Erin Abner

USA

Esharenana E. Adomi

Nigeria

Julie Agel

USA

Arthur Allignol

Germany

Margaret Allman-Farinelli

Australia

Heather Allore

USA

Ming-Wen An

USA

Viola Angelini

Netherlands

Kristopher Attwood

USA

Matthew Baldwin

USA

Heejung Bang

USA

Adrian Barnett

Australia

Huiman Barnhart

USA
Eric Barrette

USA

Jonathan Bartlett

UK

Heather Battles

New Zealand

Mickael Bech

Denmark

Melanie Bell

USA

Sally Bell-Syer

UK

Eran Bendavid

USA

Ralf Bender

Germany

Vance Berger

USA

Nancy Berkman

USA

Regina Bernal

Brazil

Boris Bershadsky

USA

Sahul Bharti

India

\author{
Akbar Biglarian \\ Iran \\ Evdokia Billis \\ Greece \\ Harald Binder \\ Germany \\ Ingrid Binswanger \\ USA
}

Ann Blanc

USA

Paul Blanche

Denmark

Emily Blood

USA

Ben Bolker

Canada

Lyndal Bond

Australia

Billie Bonevski

Australia

Isabelle Boutron

France

Ulisses Braga-Neto

USA

Ruta Brazauskas

USA

Correspondence: giulia.mangiameli@biomedcentral.com

BioMed Central, Floor 6, 236 Gray's Inn Road, London WC1X 8HB, UK 
Ann Bi Bremander

Sweden

Frank Bretz

Switzerland

Britton Brewer

USA

Camille Brisset

Canada

Nicky Britten

UK

Maarten Buis

Germany

Helen Burchett

UK

Alex Burdorf

Netherlands

Paul-Christian Bürkner

Germany

Elaine Burns

UK

Steve Buyske

USA

Julio Cabrero-Garcia

Spain

Chunyan Cai

USA

Lisa Calderwood

UK

Jerrilyn Cambron

USA

John Cape

UK

John Carlisle

UK

Bernie Carter

UK

Kristie Carter

New Zealand

Susan Cassels

USA

Ya-Mei Chang

Taiwan

Anne Chao

Taiwan
Yiyi Chen

USA

Chyong-Mei Chen

Taiwan

Hua Yun Chen

USA

Maggie Chen

Canada

Shuo Chen

USA

Xiwei Chen

USA

Zhuo Chen

USA

Sylvie Chevret

France

Chin-Tsang Chiang

Taiwan

Sangbum Choi

USA

Miew Keen Choong

Australia

Jody Ciolino

USA

Jan Clement

USA

Nancy Cook

USA

Giuliana Cortese

Italy

Susan Cox

Canada

Colin Crooks

UK

Gillian Currie

UK

Eleonora Dal Grande

Australia

Abhik Das

USA

Philippa Davies

UK

Z. John Daye

USA
Annemarie De Greeff

UK

Joris De Groot

Netherlands

Heather Dean

Canada

Thomas Debray

Netherlands

Sofia Dias

UK

Markus K. Diener

Germany

Philipp Doebler

Germany

Sarah Donegan

UK

Amylou Dueck

USA

Steven Duffy

UK

Stephanie Eckman

Germany

Solvig Ekblad

Sweden

Andrew Elders

UK

Paul B. English

USA

Diane Fairclough

USA

Libero Fatti

South Africa

Todd Favorite

USA

Lambert Felix

UK

Georg Ferber

Switzerland

Mallorie Fiero

USA

Krista Fischer

Estonia

Hans Flaatten

Norway 
Benjamin Fletcher

UK

Tamsin Ford

UK

Jan Friedrich

Canada

Alex Fu

USA

Carmen Fuentelsaz-Gallego

Spain

James Gallagher

UK

Margaret Gamalo

USA

Claudia Geue

UK

Debashis Ghosh

USA

Tracy Glass

Switzerland

Roberta Goldman

USA

Mithat Gonen

USA

Adam L Gordon

UK

Jessica Gorman

USA

Inigo Gorostiza

Spain

Sue Grady

USA

Emily Griffith

USA

Catharina Groothuis-Oudshoorn

Netherlands

Martin Gulliford

UK

Kurinchi Gurusamy

UK

Chad Gwaltney

USA

Melissa Habel

USA
Alix Hall

Bo $\mathrm{Hu}$

Australia

Gill Hall

New Zealand

Bernhard Haller

Germany

Michele Hamm

Canada

Sebastien Haneuse

USA

Anthony Hanley

Canada

Frank Harrell

USA

Katie Harron

UK

Yulei He

USA

Weili He

USA

Dick Heasman

UK

Karla Hemming

UK

Anne Hickey

Ireland

Ralf-Dieter Hilgers

Germany

Niels Henrik Hjollund

Denmark

Zoe Hoare

UK

Rebecca Holman

Netherlands

Jeremy Howick

UK

Annika Hoyer

Germany

Iztok Hozo

USA

Asbjørn Hróbjartsson

Denmark

Chiu-Hsieh (Paul) Hsu USA
USA

Yueqin $\mathrm{Hu}$

USA

Rebecca Hubbard

USA

Carmel Hughes

UK

Matthew Hutmacher

USA

Thao Huynh

Canada

Grace Irimu

Kenya

Dan Jackson

UK

Zeb Jamrozik

Australia

Zhenyu Jia

USA

Zhen Jiang

USA

Benjamin Johns

USA

Grace Johnston

Canada

Shahab Jolani

Netherlands

Galen Joseph

USA

Janet Jull

Canada

Marie-Laure Kaiser

Switzerland

Zeynal Karaca

USA

Ghassan Karam

Switzerland

Juha Karvanen

Finland

Benjamin Kasenda

Switzerland

Monika Kastner

Canada 
Jacob Kean

USA

Heather Keller

Canada

Joanna Kesten

UK

Diba Khan

USA

Jane Kim

USA

Jonathan Kimmelman

Canada

Shiho Kino

Japan

Maria Kleinstaeuber

Germany

Stefanie Klug

Germany

Guido Knapp

Germany

Masha Kocherginsky

USA

Ruwanthi Kolamunnage Dona

UK

Maiying Kong

USA

Jochem König

Germany

Daniel Kotz

Netherlands

Bernd Kowall

Germany

Walter Kremers

USA

David Kriebel

USA

Judith Kulig

Canada

Kalyanaraman Kumaran

UK

Karen Kuntz

USA

Raymond Kuo

Taiwan
Emil Kupek

Brazil

Oliver Kuss

Germany

Lucie Laflamme

Sweden

Peter Lane

UK

Camille Lassale

UK

Nicholas Latimer

UK

Anders Ledberg

Sweden

Chi Hyun Lee

USA

Pak Kuen Philip Lee

Hong Kong

Sunghee Lee

USA

Mariska M. G. Leeflang

Netherlands

Catherine Legrand

Belgium

Sam Lendle

USA

Liang $\mathbf{L i}$

USA

Ruosha Li

USA

Dan Liao

USA

Lawrence Lin

USA

Feng-Chang Lin

USA

Melissa Lindeman

Australia

Marie Linder

Sweden

Aiyi Liu

USA

Jordan Louviere

Australia
Thomas Love

USA

Guobing Lu

UK

Qing Lu

USA

Jay Lubin

USA

Sonja Lumme

Finland

Nan Luo

Singapore

Sheng Luo

USA

Theodore Lystig

USA

Joy Macdermid

Canada

Andrew Mackinnon

Australia

Laura Macpherson

USA

Susan Magasi

USA

Anselm Mak

Singapore

Donald Malec

USA

Mario Malicki

Croatia

Judith Malmgren

USA

Ian Marschner

Australia

Dimitris Mavridis

Greece

Nancy Mayo

Canada

Jim Mccambridge

UK

Elaine Mccoll

UK

Richard Mceachin

USA 


\begin{tabular}{|c|c|c|}
\hline $\begin{array}{l}\text { Lorcan Mcgarvey } \\
\text { UK }\end{array}$ & $\begin{array}{l}\text { Diana Ngo } \\
\text { USA }\end{array}$ & $\begin{array}{l}\text { Petros Pechlivanoglou } \\
\text { Canada }\end{array}$ \\
\hline $\begin{array}{l}\text { Kevin Mcgeechan } \\
\text { Australia }\end{array}$ & $\begin{array}{l}\text { Pentti Nieminen } \\
\text { Finland }\end{array}$ & $\begin{array}{l}\text { Michael Pencina } \\
\text { USA }\end{array}$ \\
\hline $\begin{array}{l}\text { David Mclernon } \\
\text { UK }\end{array}$ & $\begin{array}{l}\text { Theo Niyonsenga } \\
\text { Australia }\end{array}$ & $\begin{array}{l}\text { Lisa Pennells } \\
\text { UK }\end{array}$ \\
\hline $\begin{array}{l}\text { Timothy Mcmurry } \\
\text { USA }\end{array}$ & $\begin{array}{l}\text { Francis Obare } \\
\text { Kenya }\end{array}$ & $\begin{array}{l}\text { Hans-Peter Piepho } \\
\text { Germany }\end{array}$ \\
\hline $\begin{array}{l}\text { Florian Meinfelder } \\
\text { Germany }\end{array}$ & $\begin{array}{l}\text { Toshiyuki Ojima } \\
\text { Japan }\end{array}$ & $\begin{array}{l}\text { Carol Pierannunzi } \\
\text { USA }\end{array}$ \\
\hline $\begin{array}{l}\text { Joris Menten } \\
\text { Belgium }\end{array}$ & $\begin{array}{l}\text { Roberto S. Oliveri } \\
\text { Denmark }\end{array}$ & $\begin{array}{l}\text { Sabrina Pit } \\
\text { Australia }\end{array}$ \\
\hline $\begin{array}{l}\text { Lisa Merry } \\
\text { Canada }\end{array}$ & $\begin{array}{l}\text { Rumana Omar } \\
\text { UK }\end{array}$ & $\begin{array}{l}\text { Maja Pohar Perme } \\
\text { Slovenia }\end{array}$ \\
\hline $\begin{array}{l}\text { Susan Michie } \\
\text { UK }\end{array}$ & $\begin{array}{l}\text { Igho Onakpoya } \\
\text { UK }\end{array}$ & $\begin{array}{l}\text { Gregory Pond } \\
\text { Canada }\end{array}$ \\
\hline $\begin{array}{l}\text { Mark Mikkelsen } \\
\text { USA }\end{array}$ & $\begin{array}{l}\text { Dermot O'Reilly } \\
\text { UK }\end{array}$ & $\begin{array}{l}\text { Diana Porro-Munoz } \\
\text { Italy }\end{array}$ \\
\hline $\begin{array}{l}\text { Andrew Miles } \\
\text { UK }\end{array}$ & $\begin{array}{l}\text { Matthew Page } \\
\text { Australia }\end{array}$ & $\begin{array}{l}\text { Corinna Porteri } \\
\text { Italy }\end{array}$ \\
\hline $\begin{array}{l}\text { Peter Molenaar } \\
\text { USA }\end{array}$ & $\begin{array}{l}\text { Qing Pan } \\
\text { USA }\end{array}$ & $\begin{array}{l}\text { George Potamias } \\
\text { Greece }\end{array}$ \\
\hline $\begin{array}{l}\text { David Morgan } \\
\text { USA }\end{array}$ & $\begin{array}{l}\text { Nikolaos Pandis } \\
\text { Switzerland }\end{array}$ & $\begin{array}{l}\text { Matthew Powney } \\
\text { UK }\end{array}$ \\
\hline $\begin{array}{l}\text { Megan Morris } \\
\text { USA }\end{array}$ & $\begin{array}{l}\text { Xavier Paoletti } \\
\text { France }\end{array}$ & $\begin{array}{l}\text { Justin Presseau } \\
\text { UK }\end{array}$ \\
\hline $\begin{array}{l}\text { Tim Morris } \\
\text { UK }\end{array}$ & $\begin{array}{l}\text { Spyridon Papageorgiou } \\
\text { Germany }\end{array}$ & $\begin{array}{l}\text { David Prieto-Merino } \\
\text { UK }\end{array}$ \\
\hline $\begin{array}{l}\text { Richard Moser } \\
\text { USA }\end{array}$ & $\begin{array}{l}\text { María Carmen Pardo } \\
\text { Spain }\end{array}$ & $\begin{array}{l}\text { Paolo Emilio Puddu } \\
\text { Italy }\end{array}$ \\
\hline $\begin{array}{l}\text { Deborah Mullen } \\
\text { USA }\end{array}$ & $\begin{array}{l}\text { Richard Parker } \\
\text { UK }\end{array}$ & $\begin{array}{l}\text { Guoyou Qin } \\
\text { China }\end{array}$ \\
\hline $\begin{array}{l}\text { Katharina Müller } \\
\text { Germany }\end{array}$ & $\begin{array}{l}\text { Lianne Parkin } \\
\text { New Zealand }\end{array}$ & $\begin{array}{l}\text { Tielin Qin } \\
\text { USA }\end{array}$ \\
\hline $\begin{array}{l}\text { Zachary Munn } \\
\text { Australia }\end{array}$ & $\begin{array}{l}\text { Eunice Park-Lee } \\
\text { USA }\end{array}$ & $\begin{array}{l}\text { Hude Quan } \\
\text { Canada }\end{array}$ \\
\hline $\begin{array}{l}\text { Maureen Murdoch } \\
\text { USA }\end{array}$ & $\begin{array}{l}\text { Nick Parsons } \\
\text { UK }\end{array}$ & $\begin{array}{l}\text { Gillian Raab } \\
\text { UK }\end{array}$ \\
\hline $\begin{array}{l}\text { Saman Muthukumarana } \\
\text { Canada }\end{array}$ & $\begin{array}{l}\text { Janet Parsons } \\
\text { Canada }\end{array}$ & $\begin{array}{l}\text { Tamara Rader } \\
\text { Canada }\end{array}$ \\
\hline $\begin{array}{l}\text { Duncan Neuhauser } \\
\text { USA }\end{array}$ & $\begin{array}{l}\text { Lisa Pastore } \\
\text { USA }\end{array}$ & $\begin{array}{l}\text { Shafiqur Rahman } \\
\text { UK }\end{array}$ \\
\hline $\begin{array}{l}\text { Hannelore Neuhauser } \\
\text { Germany }\end{array}$ & $\begin{array}{l}\text { Sujata Patil } \\
\text { USA }\end{array}$ & $\begin{array}{l}\text { Angelique Ralph } \\
\text { Australia }\end{array}$ \\
\hline
\end{tabular}


Geraldine Rauch

Germany

David Reeves

UK

Joahnnes Reitsma

Netherlands

Matthew Ridd

UK

Suzan Robroek

Netherlands

José Manuel Rodriguez Barrios

Spain

Kris Rogers

Australia

Patricia Rogers

Australia

\section{Aldo Rosano}

Italy

Gerta Rücker

Germany

Lila Rutten

USA

Euijung Ryu

USA

Shubhayu Saha

USA

Tolulope Sajobi

Canada

Joseph Sakshaug

Germany

Margaret Sampson

Canada

Nancy Santesso

Canada

Jonathan Schildcrout

USA

Christopher Schmid

USA

Rainer Schnell

Germany

Shaun Scholes

UK

Ellen Schultz

USA
Heinzpeter Schwermer

Switzerland

Frederic Selck

USA

Stephen Senn

Luxembourg

Joseph Sewell Araya

Costa Rica

Mike Sharland

UK

Paul Shekelle

USA

Tsung-Jen Shen

Taiwan

Irina Shevtsova

Russian Federation

Mohamed Shoukri

Saudi Arabia

Juned Siddique

USA

Becky Skidmore

Canada

Rob Smith

USA

David Smith

USA

Mitchell Smith

Australia

Niels Smits

Netherlands

Kym Snell

UK

Harriet Sommer

Germany

Fujian Song

UK

Maria Alice Souza De Oliveira Dode Brazil

Bart Spiessens

Belgium

Cristian Spitoni

Netherlands

James Stamey

USA
Renee Stark

Germany

Ewout Steyerberg

Netherlands

Manfred Stommel

USA

Hong Sun

China

Joshua Swift

USA

Nian-Sheng Tang

China

Ge Tao

USA

Parisa Tehranifar

USA

Armando Teixeira-Pinto

Australia

Lucy Thairu

USA

James Thomas

UK

Daniel Thorburn

Sweden

Kristian Thorlund

Canada

Andrea Troxel

USA

Apostolos Tsiachristas

Netherlands

Erick Turner

USA

Werner Vach

Germany

Linda Valeri

USA

Stef Van Buuren

Netherlands

Ben Van Calster

Belgium

Johanna Van Der Bom

Netherlands

Danielle Van Der Windt

UK 


\begin{tabular}{|c|c|c|}
\hline Emily Van Meter & Evelyn Whitlock & Jun Yang \\
\hline USA & USA & China \\
\hline $\begin{array}{l}\text { Gert Van Valkenhoef } \\
\text { Netherlands }\end{array}$ & $\begin{array}{l}\text { Marie Wiberg } \\
\text { Sweden }\end{array}$ & $\begin{array}{l}\text { Wen Ye } \\
\text { USA }\end{array}$ \\
\hline $\begin{array}{l}\text { Vassilis Vasdekis } \\
\text { Greece }\end{array}$ & $\begin{array}{l}\text { Andreas Wienke } \\
\text { Germany }\end{array}$ & $\begin{array}{l}\text { Zhiying You } \\
\text { USA }\end{array}$ \\
\hline $\begin{array}{l}\text { Monica Vasquez } \\
\text { USA }\end{array}$ & $\begin{array}{l}\text { Andrew Williams } \\
\text { USA }\end{array}$ & $\begin{array}{l}\text { Tracey Young } \\
\text { UK }\end{array}$ \\
\hline $\begin{array}{l}\text { Jan Verbakel } \\
\text { Belgium }\end{array}$ & $\begin{array}{l}\text { Don Willison } \\
\text { Canada }\end{array}$ & $\begin{array}{l}\text { Recai Yucel } \\
\text { USA }\end{array}$ \\
\hline $\begin{array}{l}\text { Areti Angeliki Veroniki } \\
\text { Canada }\end{array}$ & $\begin{array}{l}\text { Ed Wilson } \\
\text { UK }\end{array}$ & $\begin{array}{l}\text { Antonia Zapf } \\
\text { Germany }\end{array}$ \\
\hline $\begin{array}{l}\text { Georgia Verropoulou } \\
\text { Greece }\end{array}$ & $\begin{array}{l}\text { Carlos King Ho Wong } \\
\text { Hong Kong }\end{array}$ & $\begin{array}{l}\text { Alan Zaslavsky } \\
\text { USA }\end{array}$ \\
\hline $\begin{array}{l}\text { Andrew Vickers } \\
\text { USA }\end{array}$ & $\begin{array}{l}\text { Alex Wong } \\
\text { USA }\end{array}$ & $\begin{array}{l}\text { Nikolajs Zeps } \\
\text { Australia }\end{array}$ \\
\hline $\begin{array}{l}\text { Shankar Viswanathan } \\
\text { USA }\end{array}$ & $\begin{array}{l}\text { Lesley Wood } \\
\text { UK }\end{array}$ & $\begin{array}{l}\text { Han Zhang } \\
\text { USA }\end{array}$ \\
\hline $\begin{array}{l}\text { Monique Margaretha Jozefa Walenkamp } \\
\text { Netherlands }\end{array}$ & $\begin{array}{l}\text { Beth Woods } \\
\text { UK }\end{array}$ & $\begin{array}{l}\text { Xu Zhang } \\
\text { USA }\end{array}$ \\
\hline $\begin{array}{l}\text { Muhammad Walji } \\
\text { USA }\end{array}$ & $\begin{array}{l}\text { Abera Wouhib } \\
\text { USA }\end{array}$ & $\begin{array}{l}\text { Guangyu Zhang } \\
\text { USA }\end{array}$ \\
\hline $\begin{array}{l}\text { Stephen John Walters } \\
\text { UK }\end{array}$ & $\begin{array}{l}\text { Kath Wright } \\
\text { UK }\end{array}$ & $\begin{array}{l}\text { Yang Zhao } \\
\text { USA }\end{array}$ \\
\hline $\begin{array}{l}\text { Pengyuan Wang } \\
\text { USA }\end{array}$ & $\begin{array}{l}\text { Changchun Xie } \\
\text { USA }\end{array}$ & $\begin{array}{l}\text { Wenle Zhao } \\
\text { USA }\end{array}$ \\
\hline $\begin{array}{l}\text { Ming Wang } \\
\text { USA }\end{array}$ & $\begin{array}{l}\text { Tu Xu } \\
\text { USA }\end{array}$ & $\begin{array}{l}\text { Jin Zhou } \\
\text { USA }\end{array}$ \\
\hline $\begin{array}{l}\text { Sijian Wang } \\
\text { USA }\end{array}$ & $\begin{array}{l}\text { Pengcheng Xun } \\
\text { USA }\end{array}$ & $\begin{array}{l}\text { Muhan Zhou } \\
\text { USA }\end{array}$ \\
\hline $\begin{array}{l}\text { Mark Weiner } \\
\text { USA }\end{array}$ & $\begin{array}{l}\text { Kirsten Yaffe } \\
\text { USA }\end{array}$ & $\begin{array}{l}\text { Yijie Zhou } \\
\text { USA }\end{array}$ \\
\hline $\begin{array}{l}\text { Edie Weller } \\
\text { USA }\end{array}$ & $\begin{array}{l}\text { Hsin-Chou Yang } \\
\text { Taiwan }\end{array}$ & $\begin{array}{l}\text { Ying Zhou } \\
\text { USA }\end{array}$ \\
\hline $\begin{array}{l}\text { Shih-Feng Weng } \\
\text { Taiwan }\end{array}$ & $\begin{array}{l}\text { Jichen Yang } \\
\text { USA }\end{array}$ & $\begin{array}{l}\text { Jihao Zhou } \\
\text { USA }\end{array}$ \\
\hline $\begin{array}{l}\text { David Wheeler } \\
\text { USA }\end{array}$ & $\begin{array}{l}\text { Min Yang } \\
\text { USA }\end{array}$ & $\begin{array}{l}\text { Ming Zhu } \\
\text { USA }\end{array}$ \\
\hline
\end{tabular}

